\title{
Totally Laparoscopic Pancreaticoduodenectomy for Pancreatic Head Cancer with Involvement of the Superior Mesenteric Vein-Portal Vein Confluence
}

\author{
Ziad T. Awad, MD, FACS
}

Division of Minimally Invasive Surgery, Department of Surgery, University of Florida College of Medicine - Jacksonville, Jacksonville, FL

\begin{abstract}
Laparoscopic pancreaticoduodenectomy is a technically demanding procedure. In this video, we demonstrate the technical aspects of performing the procedure. In a 50-year-old male with ascending cholangitis, endoscopic retrograde cholangiopancreatography was unsuccessful, and percutaneous transhepatic cholangiography was carried out for biliary decompression. Endoscopic ultrasound plus fine-needle aspiration showed pancreatic head adenocarcinoma. The procedure was carried out using five trocars, and extensive lymphadenectomy was undertaken. The uncinate process was skeletonized off the superior mesenteric artery. The right lateral aspect of the superior mesenteric vein-portal vein confluence was
\end{abstract}

involved with the cancer. The laparoscopic linear stapler was used to transect part of the vein en bloc with the specimen. All margins were negative and all the anastomoses were done using laparoscopic intracorporeal suturing. Operative time was $8 \mathrm{~h} 20 \mathrm{~min}$, and hospital stay was 5 days. Final pathology was T3 N1 (one lymph node out of 40 was positive).

Conclusion. Laparoscopic pancreaticoduodenectomy can be performed safely in selected cases of pancreatic head cancer with vascular involvement. Skilled laparoscopic skills are necessary to execute such procedures safely.
Electronic supplementary material The online version of this article (doi:10.1245/s10434-014-3736-0) contains supplementary material, which is available to authorized users.

(C) Society of Surgical Oncology 2014

First Received: 11 March 2014;

Published Online: 20 May 2014

Z. T. Awad, MD, FACS

e-mail: ziad.awad@jax.ufl.edu 